\title{
Radiotherapy: Changing the Game in Immunotherapy
}

Sandra Demaria ${ }^{1,3}$, C. Norman Coleman ${ }^{2}$, Silvia C. Formenti ${ }^{1}$

${ }^{1}$ Department of Radiation Oncology, Weill Cornell Medicine, 1300 York Ave, Box 169,

New York, NY 10065; ${ }^{2}$ Radiation Oncology Branch, Center for Cancer Research and

Radiation Research Program, Division of Cancer Treatment and Diagnosis, National

Cancer Institute, NIH, Bethesda, MD.

${ }^{3}$ Corresponding author: Demaria, S. (szd3005@med.cornell.edu) 


\begin{abstract}
Immune checkpoint inhibitors are effective in cancer treatment. A pre-existing immune response demonstrated by significant pretreatment tumor lymphocytic infiltration is a pre-requisite for response. Within such infiltrated tumors, referred as "hot", immune checkpoint inhibitors rescue anti-tumor T cells activity. In contrast, "cold" tumors lack lymphocytic infiltration and are refractory to immunotherapy. Preclinical data show that radiotherapy sensitizes refractory tumors to immune checkpoint inhibitors by recruiting anti-tumor T cells. Despite the growing number of clinical studies testing radiation's ability to enhance immunotherapy, clinical evidence that it converts cold tumors into responsive ones remains elusive. Here we review evidence that radiotherapy is not only an occasional enhancer of immunotherapy's effects but a "game changer", and propose a blueprint to test this.
\end{abstract}




\section{The promise of radiotherapy as a partner for immunotherapy.}

Ionizing radiation alone or combined with systemic therapy is a highly effective treatment for local tumor control. Research to improve responses to radiation has advanced the understanding of radiation-induced damage and repair mechanisms in neoplastic and normal cells [1]. In addition, technological improvements allow optimal dose delivery to the tumor while sparing adjacent structures [2]. The tumor microenvironment (TME), particularly the degree of oxygenation, has been a longstanding focus of radiation research. Recently, increased appreciation for the complex TME role in therapeutic response has broadened radiation biology research to include the effects of radiation on tumor vasculature, fibroblasts and immune cells [3].

In concert, cancer immunology has seen tremendous progress, leading to the development of immunotherapies [4]. The critical role of the immune system from cancer development to metastasis has been dissected in experimental models [5] and supported by growing evidence from human studies $[6,7]$. Together, these data provide the basis for a novel functional classification of human tumors based on their immunological phenotype $[8,9]$. In immune competent hosts all tumors evolve under the pressure of the immune system, which recognizes stress signals and altered cellular phenotypes resulting from neoplastic transformation and can eliminate the aberrant cells. Tumor progression requires the cancer cells to develop resistance to immune attack. Immune escape mechanisms are complex, but the resulting tumor phenotypes can be broadly separated into two main groups, based on rich or poor infiltration of $\mathrm{T}$ 
lymphocytes, often referred to as "hot" and "cold" tumors, respectively $[10,11]$. In hot tumors the relative proportion of effector CD8 and regulatory $\mathrm{T}$ cells varies, reflecting different degrees of immunosuppression and influencing the propensity of a tumor to progress [7]. However, this definition is important in that the most significant success in immunotherapy has been obtained with treatments that block immune suppressive pathways. These treatments remove the brakes that prevent an ongoing immune response from rejecting the tumor, but are ineffective in patients without such a pre-existing response [11-13]. Unfortunately, at the time of cancer diagnosis cold tumors constitute the most frequent phenotype encountered among solid tumors. This may explain the current "ceiling" of response rates achievable with immune checkpoint inhibitors (ICI), generally in the range of $20-40 \%$.

Strategies to extend ICI benefits to more patients are clearly warranted and the identification of combination treatments converting cold tumors into hot ones is an active area of research [10,14] (Figure 1, Key Figure). Local radiotherapy has emerged as a front-runner strategy, following pre-clinical demonstration that it can convert tumors unresponsive to anti-CTLA-4 into responsive ones $[15,16]$, and also from several clinical observations of responses in patients treated with the anti-CTLA-4 antibody ipilimumab that were seen only after radiation treatment [17-20]. Most importantly, local radiotherapy applied to a single lesion was able to induce responsiveness to anti-CTLA-4 in non-irradiated metastases, suggesting that irradiation of one site can jump-start a systemic anti-tumor immune response. This has spurred research to investigate the pro- 
immunogenic effects of radiation, a treatment traditionally considered as being immune suppressive [21, 22].

Following the clinical success of antibodies targeting the PD-1/PDL-1 pathway in multiple tumor types [23], there has been widespread interest in testing these agents in combination with radiation. Results from pre-clinical studies have demonstrated enhanced effects between radiation and anti-PD-1 or anti-PDL-1 [24-26], and clinical trials are in progress.

Thus, while a benefit of radiation treatment during immunotherapy has been observed, it remains to be determined if and how radiotherapy enhances the response to "hot" tumors and converts a subset of tumors from unresponsive to responsive. Radiotherapy is unique as a partner for combination treatments in that it is readily available, unrestricted by patent rights, and part of the standard of care for almost every cancer. These advantages may also be a curse: because of its easy access, radiotherapy can be applied as a simple add-on to any immunotherapy, as suggested by the flurry of studies initiated in the US and abroad, without needed considerations about dose, fractionation, sequencing and timing. Without bench-to-bedside research that focuses on molecular changes from response to radiation, including how radiation interacts with the unique microenvironment of different tumors, these trials risk achieving inconclusive results. Here we critically review evidence that radiotherapy could be a "game changer" in addition to an occasional enhancer of immunotherapy's effects, and propose a blueprint to establish the role of radiotherapy in immunotherapy. 


\section{Radiation as a generator of anti-tumor $T$ cells: beyond "proof of principle".}

The contribution of $\mathrm{T}$ cells to radiation-induced tumor control was shown in mouse models over thirty years ago [27] and, more recently, the availability of $\mathrm{T}$ cell receptor (TCR)-transgenic mice made it possible to unequivocally demonstrate that radiation can induce priming of T cells to exogenous "model" antigens expressed by tumors [28, 29]. These studies, together with the demonstration that radiation induces immunogenic cell death $[30,31]$, have provided proof of principle evidence that radiation can induce tumorspecific T cells. However, one caveat of experimental models is the fact that transplantable tumors expressing relatively high levels of strong exogenous antigens (e.g., ovalbumin) are immunogenic and generate some degree of spontaneous anti-tumor $\mathrm{T}$ cell responses. Therefore, they do not adequately model primary cold human tumors.

Priming of spontaneous anti-tumor $\mathrm{T}$ cell responses in immunogenic tumors has been recently shown to be mediated by a $\mathrm{CD} 8 \alpha^{+} \mathrm{CD} 103^{+}$tumor-infiltrating dendritic cell (DC) subset that is activated by autocrine production of interferon (IFN) $\beta$ when DC sense cancer cell-derived DNA via the cGAS-STING pathway [32]. Importantly, activation of this pathway can be enhanced by tumor irradiation [33]. In contrast, $T$ cell priming to endogenous antigens expressed by two poorly immunogenic mouse tumors was not achieved with radiation alone. Although activation of the STING pathway was not analyzed in this study, transforming growth factor (TGF) $\beta$ was shown to hinder 
activation of DCs in the irradiated tumor [34]. These data suggest that radiation has a limited ability to overcome the barriers to T cell priming in tumors that more closely resemble cold tumors, where additional inhibitors of immunosuppression may be needed. The development of new mouse models mimicking the molecular changes identified in subsets of cold human tumors, such as the recently reported melanoma model lacking intratumoral $\mathrm{CD} 8 \alpha^{+} \mathrm{CD}_{103}{ }^{+} \mathrm{DC}$ [35], will be required to provide much needed information about the ability of radiation to prime anti-tumor T cells to immunotherapyresistant tumors that use various immune-escape pathways.

\section{Radiation dose and fractionation: not a "one size fits all" solution}

Most pre-clinical studies examining the immunogenic effects of radiation alone or in combination with immunotherapy have been limited to testing a single radiation dose or radiation regimen, despite the fact that radiation given as a single dose to human cancer cells induces markedly different gene expression profiles when compared to radiation given in multiple fractions, in vitro and in vivo [36, 37]. Multiple variables affect the outcome of radiation treatment in mouse models: differences in intrinsic cancer cell radiosensitivity and immunogenicity; expression of exogenous proteins; time from initial cancer cell implantation to allow for the establishment of a more immunosuppressive and hypoxic tumor microenvironment at the time of radiation; or implantation site (subcutaneous vs orthotopic) (BOX 1). Thus, information gathered from pre-clinical studies should not be interpreted as providing generalizable evidence applicable to clinical trial design without taking into consideration the influence of the above factors. 
In addition, few studies have tested the ability of radiation to induce an abscopal effect, a key clinically relevant endpoint $[38,39]$. Despite the limitations, side-by-side testing of different radiation regimens in the same tumor and treatment model allowed to compare the ability of three radiation regimens to induce abscopal effects in combination with anti-CTLA-4. A single 20 Gy dose was ineffective, while 6 Gy given $5 \mathrm{X}$ and 8 Gy given $3 \mathrm{X}$ achieved partial and complete abscopal responses in the mice [16]. The potential translational relevance of these findings is suggested by the fact that abscopal responses reported in patients treated with anti-CTLA-4 were seen using radiation regimens similar to those effective in mice $[17,20,40]$.

The choice of radiation dose depends on the effect that needs to be achieved to facilitate tumor rejection. For example, preclinical data indicated that low dose radiation $(0.5$ to 2 Gy) was effective for reprogramming tumor-associated macrophages (TAM) and for vascular normalization [41]. Thus, low single dose radiation could be the sufficient in situations where the so called "vascular barrier" is the main obstacle to T cell infiltration into the tumor and anti-tumor T cells are elicited by vaccination or are adoptively transferred [42].

Overall, more mechanistic information about how dose and fractionation influence the interaction of radiation with the pre-existing TME is needed and can be obtained using the expanding arrays of tumor models available (BOX 2). Rigorous mechanistic information will inform a rational rather than an empirical choice of radiation dose and fractionation to use in combination with immunotherapy in the clinic. 


\section{Lessons learned from clinical trials}

Due to the accelerating pace at which immunotherapies are becoming standard of care for a growing number of cancers it is not unusual for radiation to be used at some point in the treatment of patients who do not respond or respond poorly to immunotherapy.

Retrospective analyses have attempted to assess the frequency of abscopal effects in this setting [43-45]. However, specifically designed trials are needed to make conclusions about the role of radiotherapy as an adjuvant or critical component of immunotherapies. There are currently 25 trials evaluating the combination of radiation with CTLA-4 inhibitors and radiation, 20 trials testing combinations with PD-1 or PDL-1 (in clinicaltrials.gov), and more are being planned. In the absence of more information from pre-clinical work to guide the choice of radiation dose, fractionation, site of irradiation, and sequencing with each type of immunotherapy, the design of these trials is largely done using empirical considerations and, therefore, results may be inconclusive or fail to demonstrate the ability of radiation to synergize with immunotherapy.

Two clinical trials are presented as examples. 1) In a phase III trial, 799 patients with castrate-resistant metastatic prostate cancer that progressed after docetaxel chemotherapy were randomized to receive a palliative single 8 Gy dose to a bone lesion only, or radiation combined with ipilimumab. There was no evidence for benefit of combined therapy [46] and, while there are many possible reasons [47], one obvious lesson is that 
the radiation dose and target were inadequate to generate an in situ vaccine. 2) In a phase I study of 22 patients with metastatic melanoma treated with radiation and ipilimumab, $18 \%$ of patients had partial response (best outcome) and $18 \%$ showed stable disease in non-irradiated lesions [48]. This response rate is comparable to that of ipilimumab alone [49]. The trial was designed to determine the maximum tolerated radiation dose when given with ipilimumab and the lack of increased toxicities attributable to combination therapy is reassuring. However, the majority of patients may have received suboptimal doses of radiation, as suggested by the lack of control of the irradiated lesion in 4 out of 12 patients treated with radiation dose level 1 (6Gy or 8Gy given only twice). In addition, they were not selected to be resistant to ipilimumab alone, precluding any conclusion about the contribution of radiation in eliciting a systemic response. The delayed administration of ipilimumab, starting 3 to 5 days after radiation, does not translate the best scheduling defined by pre-clinical data or clinical observations of abscopal responses $[16,17,43]$, factors that may have contributed to the lack of a clear indication that radiation improved responses to ipilimumab. The study also demonstrates the importance of correlative studies to help understand the potential role of radiation in converting unresponsive patients with cold tumors into responders. While the study did not include measurements of tumor-specific immune responses in peripheral blood, pre-treatment tumor tissue was available from 12 of 22 patients and PDL-1 expression was detected in 4 out of 9 non-responding patients but in none of the two responding patients tested. PDL-1 is up-regulated in the presence of an immune-active tumor microenvironment but its expression can also be driven by oncogenic pathways and/or hypoxia [50]. Therefore, results concerning PDL-1 coming from this study are difficult to interpret and help little 
to guide the design of future studies. Based on data from a pre-clinical mouse melanoma model, the authors advocated the combined inhibition of CTLA-4 and PD-1 with radiation [48]. The combination of these ICI has achieved responses in $61 \%$ of BRAF wild-type melanomas [51], thus trials to demonstrate the additional benefits of radiation will need to be carefully crafted.

\section{Integrating pre-clinical models with clinical studies: the quest for more evidence}

Mutation-generated neoantigens are unique to each tumor and were recently shown to be targets of effective tumor rejection in patients [52-54]. As proposed by Schumacher and Schreiber, radiation could be a shortcut to elicit T cell responses to neoantigens [55]. However, evidence that radiation can reliably achieve this effect is not yet available. Interestingly, radiation was shown to induce $\mathrm{T}$ cell responses to peptides derived from proteins up-regulated in response to radiation [56], suggesting that radiation could potentially expose antigenic mutations otherwise silent in non-irradiated tumors. This could be extremely valuable in the setting of locally advanced cancers where preoperative radiation and immunotherapy might improve the rate of complete pathological response. In this setting, irradiation of draining lymph nodes, which are often treated, should be avoided to allow for priming of T cells. Immunotherapy plus radiation might also have a role in brain tumors by means of achieving tumor control without resorting to high radiation doses and thereby avoiding the risk of radionecrosis. 
For metastatic disease the goal is to achieve both local and systemic effects. While abscopal responses have been demonstrated in mice and patients [16, 17, 20, 57], it is unknown if the antigenic targets of $\mathrm{T}$ cell-mediated rejection are shared by the irradiated and non-irradiated metastases. Besides the radiation-specific epitopes described above, modulation of multiple surface molecules facilitates recognition of irradiated tumor cells by $\mathrm{T}$ cells [58], raising the concern that epitopes present in lower abundance or of low affinity for the TCR may not engage T cells in non-irradiated tumors. The differential specificity of $\mathrm{T}$ cells that reject irradiated and non-irradiated tumors may not be a concern if there are multiple antigenic targets, including some that are common between the irradiated tumor and other metastatic lesions. There is some evidence that radiation can broaden the numbers of $\mathrm{T}$ cell clones in the tumor, consistent with induction of a polyvalent response $[48,59]$. However, it is unknown if the antigenic specificity of $\mathrm{T}$ cells primed by radiation in the presence of antibodies blocking CTLA-4 or PD-1, or both, is qualitatively different. Such possibility is likely, given the different role of CTLA-4, which is engaged by co-stimulatory molecules expressed on antigen-presenting cells during $\mathrm{T}$ cell activation, and PD-1, which is predominantly engaged by PDL-1 expressed on the cancer cells during the effector phase [11]. The latter question is important because it can provide the rationale for best sequencing these treatments in clinical trials.

\section{Clinical trial design: how to demonstrate the contribution of radiation to immunotherapy response?}


Studying the use of radiation as an adjuvant to immunotherapy presents several challenges. Assessing efficacy of immune modulation triggered by radiation will likely require different endpoints than for radiation therapy alone. A similar situation occurred when cyclophosphamide was repurposed as an immune modulator and was used at doses lower than those required for its cytotoxic effects [60]. An important consideration in the choice of radiation regimen and technique is the risk of inducing significant and prolonged lymphopenia [61]. The mean dose of radiation to the circulating lymphocyte pool is dependent on the number of radiation fractions and the volume of tissue irradiated, especially in the brain and other highly vascularized organs [62]. Stereotactic delivery of larger doses per fraction, in fewer fractions to smaller tissue volumes induced less lymphopenia and better survival in pancreatic cancer patients than the standard delivery of small doses per fraction over several weeks [63]. A provocative question is whether irradiation of only part of a tumor is sufficient if the main purpose of radiation is not the direct local tumor control but the induction of anti-tumor $\mathrm{T}$ cells that will then eliminate the tumor.

In combination trials, the contribution of radiation to patients's response should be clearly discernable. As exemplified in the melanoma trial discussed above [48], attributing to radiotherapy the credit for abscopal responses in the setting of independently effective immunotherapy requires a concurrent control arm of immunotherapy alone. Adding radiation at progression could obviate this problem, although delayed responses to ipilimumab can occur, complicating the interpretation of results. The demonstration of newly developed tumor-specific $\mathrm{T}$ cells after radiotherapy 
would support the contribution of radiation [17].

In malignancies known to be poorly responsive to ICI it is feasible to test the ability of radiotherapy to induce abscopal responses in a single arm study. We recently demonstrated an example of this approach with anti CTLA-4 and radiotherapy in nonsmall cell lung cancer. Of all patients that received radiation and completed four cycles of ipilimumab 33\% showed partial or complete abscopal responses, which demonstrates the efficacy of this combination regimen in this disease [40, 64]. Extensive correlatives studies are ongoing to identify candidate biomarkers.

\section{Concluding Remarks}

The use of radiotherapy for enhancing both local and systemic immunotherapy is an exciting and revolutionary concept, but much remains to be learned before it can be applied to treat patients (see outstanding questions). Given the substantial potential benefits to patients we believe that innovative studies accompanied by rigorous immune monitoring are required to make progress (BOX 3). The rapid growth of clinical investigations combining immunotherapy and radiotherapy, in the absence of rigorous hypothesis-driven trial design, may fail to truly exploit the emerging new paradigms, which could impair further development of the field. With novel immunotherapies entering clinical practice at an accelerating pace the need to understand how to best integrate radiation biology with tumor immunology to provide a solid rationale for 
clinical translation remains urgent.

\section{Acknowledgments:}

S. D. is supported by the National Institutes of Health (R01 CA201246); the Breast Cancer Research Foundation, and the Chemotherapy Foundation. S.C.F. is supported by the National Institutes of Health (R01 CA161891), the USA Department of Defense Breast Cancer Research Program (W81XWH-11-1-0530); and the Breast Cancer Research Foundation. 


\section{References}

1 Coleman, C.N., et al. (2014) Enhancing the efficacy of radiation therapy: premises, promises, and practicality. J Clin Oncol 32, 2832-2835

2 Timmerman, R.D., et al. (2014) Emergence of stereotactic body radiation therapy and its impact on current and future clinical practice. J Clin Oncol 32, 2847-2854

3 Barker, H.E., et al. (2015) The tumour microenvironment after radiotherapy: mechanisms of resistance and recurrence. Nat Rev Cancer 15, 409-425

4 Sharma, P. and Allison, J.P. (2015) Immune checkpoint targeting in cancer therapy: toward combination strategies with curative potential. Cell 161, 205-214

5 Schreiber, R.D., et al. (2011) Cancer immunoediting: integrating immunity's roles in cancer suppression and promotion. Science 331, 1565-1570

6 Galon, J., et al. (2006) Type, density, and location of immune cells within human colorectal tumors predict clinical outcome. Science 313, 1960-1964

7 Fridman, W.H., et al. (2012) The immune contexture in human tumours: impact on clinical outcome. Nat Rev Cancer 12, 298-306

8 Gajewski, T.F., et al. (2013) Innate and adaptive immune cells in the tumor microenvironment. Nat Immunol 14, 1014-1022

9 Galon, J., et al. (2013) The continuum of cancer immunosurveillance: prognostic, predictive, and mechanistic signatures. Immunity 39, 11-26

10 Gajewski, T.F. (2015) The Next Hurdle in Cancer Immunotherapy: Overcoming the Non-T-Cell-Inflamed Tumor Microenvironment. Semin Oncol 42, 663-671

11 Sharma, P. and Allison, J.P. (2015) The future of immune checkpoint therapy. Science $348,56-61$

12 Ji, R.-R., et al. (2012) An immune-active tumor microenvironment favors clinical response to ipilimumab. Cancer Immunol Immunother 61, 1019-1031

13 Tumeh, P.C., et al. (2014) PD-1 blockade induces responses by inhibiting adaptive immune resistance. Nature 515, 568-571

14 Melero, I., et al. (2015) Evolving synergistic combinations of targeted immunotherapies to combat cancer. Nat Rev Cancer 15, 457-472 
15 Demaria, S., et al. (2005) Immune-mediated inhibition of metastases following treatment with local radiation and CTLA-4 blockade in a mouse model of breast cancer. Clin Cancer Res 11, 728-734

16 Dewan, M.Z., et al. (2009) Fractionated but not single dose radiotherapy induces an immune-mediated abscopal effect when combined with anti-CTLA-4 antibody. Clin Cancer Res 15, 5379-5388

17 Postow, M.A., et al. (2012) Immunologic correlates of the abscopal effect in a patient with melanoma. N Engl J Med 366, 925-931

18 Hiniker, S.M., et al. (2012) A systemic complete response of metastatic melanoma to local radiation and immunotherapy. Transl Oncol 5, 404-407

19 Stamell, E.F., et al. (2013) The Abscopal Effect Associated With a Systemic Antimelanoma Immune Response. Int J Radiat Oncol Biol Phys 85, 293-295

20 Golden, E.B., et al. (2013) An Abscopal Response to Radiation and Ipilimumab in a Patient with Metastatic Non-Small Cell Lung Cancer Cancer Immunol Res 1, 365-372

21 Tang, C., et al. (2014) Combining radiation and immunotherapy: a new systemic therapy for solid tumors? Cancer Immunol Res 2, 831-838

22 Formenti, S.C. and Demaria, S. (2013) Combining radiotherapy and cancer immunotherapy: a paradigm shift. J Natl Cancer Inst 105, 256-265

23 Postow, M.A., et al. (2015) Immune Checkpoint Blockade in Cancer Therapy. J Clin Oncol 33, 1974-1982

24 Zeng, J., et al. (2013) Anti-PD-1 blockade and stereotactic radiation produce long-term survival in mice with intracranial gliomas. Int J Radiat Oncol Biol Phys 86, 343-349

25 Deng, L., et al. (2014) Irradiation and anti-PD-L1 treatment synergistically promote antitumor immunity in mice. J Clin Invest 124, 687-695

26 Dovedi, S.J., et al. (2014) Acquired resistance to fractionated radiotherapy can be overcome by concurrent PD-L1 blockade. Cancer Res 74, 5458-5468

27 Stone, H.B., et al. (1979) Effect of host immune capability on radiocurability and subsequent transplantability of a murine fibrosarcoma. J Natl Cancer Inst 63, 1229-1235

28 Lugade, A.A., et al. (2005) Local radiation therapy of B16 melanoma tumors increases the generation of tumor antigen-specific effector cells that traffic to the tumor. J Immunol 174, 7516-7523 
29 Lee, Y., et al. (2009) Therapeutic effects of ablative radiation on local tumor require CD8+ T cells: changing strategies for cancer treatment. Blood 114, 589-595

30 Apetoh, L., et al. (2007) Toll-like receptor 4-dependent contribution of the immune system to anticancer chemotherapy and radiotherapy. Nat Med 13, 1050-1059

31 Golden, E.B. and Apetoh, L. (2015) Radiotherapy and immunogenic cell death. Semin Radiat Oncol 25, 11-17

32 Woo, S.R., et al. (2014) STING-Dependent Cytosolic DNA Sensing Mediates Innate Immune Recognition of Immunogenic Tumors. Immunity 41, 830-842

33 Deng, L., et al. (2014) STING-Dependent Cytosolic DNA Sensing Promotes RadiationInduced Type I Interferon-Dependent Antitumor Immunity in Immunogenic Tumors. Immunity 41, 843-852

34 Vanpouille-Box, C., et al. (2015) TGF $\beta$ is a master regulator of radiation therapyinduced anti-tumor immunity. Cancer Res 75, 2232-2242

35 Spranger, S., et al. (2015) Melanoma-intrinsic $\beta$-catenin signalling prevents anti-tumour immunity. Nature 523, 231-235

36 Tsai, M.H., et al. (2007) Gene expression profiling of breast, prostate, and glioma cells following single versus fractionated doses of radiation. Cancer Res 67, 3845-3852

37 John-Aryankalayil, M., et al. (2010) Fractionated radiation therapy can induce a molecular profile for therapeutic targeting. Radiat Res 174, 446-458

38 Reynders, K., et al. (2015) The abscopal effect of local radiotherapy: using immunotherapy to make a rare event clinically relevant. Cancer Treat Rev 41, 503-510

39 Formenti, S.C. and Demaria, S. (2009) Systemic effects of local radiotherapy. Lancet Oncol 10, 718-726

40 Golden, E.B., et al. (2015) Abscopla responses in metastatic non-small cell lung cancer (NSCLC) patients treatded on a phase 2 study of combined radiation therapy and ipilimumab: evidence for the in situ vaccination hypothesis of radiation. Int J Radiat Oncol Biol Phys 93, S66-67

41 Klug, F., et al. (2013) Low-dose irradiation programs macrophage differentiation to an iNOS $^{+} / \mathrm{M} 1$ phenotype that orchestrates effective T cell immunotherapy. Cancer Cell 24, $589-602$ 
42 Lanitis, E., et al. (2015) Targeting the tumor vasculature to enhance T cell activity. Curr Opin Immunol 33, 55-63

43 Grimaldi, A.M., et al. (2014) Abscopal effects of radiotherapy on advanced melanoma patients who progressed after ipilimumab immunotherapy. Oncoimmunology 3, e28780

44 Barker, C.A. and Postow, M.A. (2014) Combinations of radiation therapy and immunotherapy for melanoma: a review of clinical outcomes. Int J Radiat Oncol Biol Phys 88, 986-997

45 Abuodeh, Y., et al. (2016) Systematic review of case reports on the abscopal effect. Curr Probl Cancer 40, 25-37

46 Kwon, E.D., et al. (2014) Ipilimumab versus placebo after radiotherapy in patients with metastatic castration-resistant prostate cancer that had progressed after docetaxel chemotherapy (CA184-043): a multicentre, randomised, double-blind, phase 3 trial. Lancet Oncol 15, 700-712

47 Golden, E.B. and Formenti, S.C. (2015) Radiation therapy and immunotherapy: growing pains. Int J Radiat Oncol Biol Phys 91, 252-254

48 Twyman-Saint Victor, C., et al. (2015) Radiation and dual checkpoint blockade activate non-redundant immune mechanisms in cancer. Nature 520, 373-377.

49 Robert, C., et al. (2011) Ipilimumab plus dacarbazine for previously untreated metastatic melanoma. N Engl J Med 364, 2517-2526

50 Smyth, M.J., et al. (2016) Combination cancer immunotherapies tailored to the tumour microenvironment. Nat Rev Clin Oncol 13, 143-158

51 Postow, M.A., et al. (2015) Nivolumab and Ipilimumab versus Ipilimumab in Untreated Melanoma. N Engl J Med 372, 2006-2017

52 Gubin, M.M., et al. (2014) Checkpoint blockade cancer immunotherapy targets tumourspecific mutant antigens. Nature 515, 577-581

53 van Rooij, N., et al. (2013) Tumor exome analysis reveals neoantigen-specific T-cell reactivity in an ipilimumab-responsive melanoma. J Clin Oncol 31, e439-442

54 Robbins, P.F., et al. (2013) Mining exomic sequencing data to identify mutated antigens recognized by adoptively transferred tumor-reactive T cells. Nat Med 19, 747-752

55 Schumacher, T.N. and Schreiber, R.D. (2015) Neoantigens in cancer immunotherapy. Science 348, 69-74 
56 Reits, E.A., et al. (2006) Radiation modulates the peptide repertoire, enhances MHC class I expression, and induces successful antitumor immunotherapy. J Exp Med 203, 12591271

57 Golden, E.B., et al. (2015) Local radiotherapy and GM-CSF in patients with metastatic solid tumors: a proof of principle trial to generate abscopal responses. Lancet Oncol 16, 795-803

58 Kwilas, A.R., et al. (2012) In the field: exploiting the untapped potential of immunogenic modulation by radiation in combination with immunotherapy for the treatment of cancer. Front Oncol 2, 104

59 Pilones, K.A., et al. (2014) Unique changes in the TCR repertoire of tumor-infiltrating lymphocytes underlie the synergy of radiotherapy with CTLA-4 blockade. J Immunother Cancer 2, P141

60 Le, D.T. and Jaffee, E.M. (2012) Regulatory T-cell modulation using cyclophosphamide in vaccine approaches: a current perspective. Cancer Res 72, 3439-3444

61 Grossman, S.A., et al. (2015) Survival in Patients With Severe Lymphopenia Following Treatment With Radiation and Chemotherapy for Newly Diagnosed Solid Tumors. J Natl Compr Canc Netw 13, 1225-1231

62 Yovino, S., et al. (2013) The etiology of treatment-related lymphopenia in patients with malignant gliomas: modeling radiation dose to circulating lymphocytes explains clinical observations and suggests methods of modifying the impact of radiation on immune cells. Cancer Invest 31, 140-144

63 Wild, A.T., et al. (2016) Lymphocyte-Sparing Effect of Stereotactic Body Radiation Therapy in Patients With Unresectable Pancreatic Cancer. Int J Radiat Oncol Biol Phys 94, 571-579

64 Zatloukal, P., et al. (2009) Randomized phase II clinical trial comparing tremelimumab (CP-675, 206) with best supportive care (BSC) following first-line platinum-based therapy in patients (pts) with advanced non-small cell lung cancer (NSCLC). J Clin Oncol 27, 8071

65 Demaria, S. and Formenti, S.C. (2012) Radiation as an immunological adjuvant: current evidence on dose and fractionation. Front Oncol 2, 153 
66 Golden, E.B., et al. (2014) Radiation fosters dose-dependent and chemotherapy-induced immunogenic cell death. . OncoImmunology 3, e28518

67 Filatenkov, A., et al. (2015) Ablative Tumor Radiation Can Change the Tumor Immune Cell Microenvironment to Induce Durable Complete Remissions. Clin Cancer Res 21, 3727-3739

68 Sanmamed, M.F., et al. (2016) Defining the optimal murine models to investigate immune checkpoint blockers and their combination with other immunotherapies. Ann Oncol Feb 23. pii: mdw041.

69 Kreiter, S., et al. (2015) Mutant MHC class II epitopes drive therapeutic immune responses to cancer. Nature 520, 692-696

70 Jinek, M., et al. (2012) A programmable dual-RNA-guided DNA endonuclease in adaptive bacterial immunity. Science 337, 816-821

71 Sanmamed, M.F., et al. (2015) Nivolumab and Urelumab Enhance Antitumor Activity of Human T Lymphocytes Engrafted in Rag2-/-IL2R rnull Immunodeficient Mice. Cancer Res 75, 3466-3478

72 Gros, A., et al. (2016) Prospective identification of neoantigen-specific lymphocytes in the peripheral blood of melanoma patients. Nat Med 22, 433-438 


\section{Figure Legend}

Key Figure 1. Converting "cold” tumors into "hot" ones. Lack of T cell infiltration may be due to absence of dendritic cells (DCs) or presence of DCs that are not activated (tolerogenic DCs) (a). In this situation, anti-tumor T cells are not generated. In other cases, anti-tumor $\mathrm{T}$ cells that are generated spontaneously or by vaccination are excluded from the tumor by the vascular barrier, which is induced by M2 tumor-associated macrophages (TAMs) (b). By inducing cancer cell death coupled with release of danger signals, radiation can promote DC activation and their migration to the tumor-draining lymph nodes (TDLN) where they activate tumor-specific T cells. Activated effector T cells then migrate to the tumor, kill cancer cells and secrete cytokines that further activate DCs and promote TAM polarization to anti-tumor M1 phenotype, converting the tumor from cold to hot (c). Low doses of radiation can also reprogram TAM towards the M1 phenotype, allowing pre-existing T cells to infiltrate the tumor. 


\section{BOX 1. Radiation dose and fractionation in pre-clinical models.}

A broad range of radiation doses has been employed in mouse models [65]. In vitro, many radiation effects, including immunogenic cell death, are dose-dependent [66].

However, in vivo the different tumor stromal compartments (fibroblasts, endothelial cells, innate and adaptive immune cells) react differently to radiation [3], which can greatly influence the shift from an immune suppressive to an immune activating tumor microenvironment (TME). Below are some key considerations:

- Hypoxia and immune suppression. Larger tumors, especially if fast growing, develop extensive areas of necrosis, which are surrounded by hypoxic areas rich in hypoxia-inducible factor (HIF)-1 $\alpha$, a driver of immunosuppression. HIF-1 $\alpha$ and lack of oxygen markedly increase radioresistance. Development of hypoxia is not equal in all tumor models, even if treated when they are the same size. On the other end, the same tumor will respond differently to radiation depending on the size. An example comes from two studies in CT26 colorectal model. In one study radiation given at 2 Gy $X 5$ when the tumors were $100-200 \mathrm{~mm}^{3}$ led to anti-tumor T cells infiltrating the tumor and producing IFN $\gamma$, which induced upregulation of PDL-1. Blockade of this molecule or its receptor PD-1 achieved complete tumor regression [26]. In the second study tumors were treated when 5-10 times larger and only a 30 Gy single dose but not 3 Gy X 10 was able to induce anti-tumor T cells [67]. Tumors regressed completely after 30 Gy but the immune response generated was not sufficient to mediate abscopal responses, although ICI were not tested. 
- Cancer-associated fibroblasts (CAFs). CAFs are phenotypically and functionally heterogeneous and can promote or inhibit tumor growth. CAFs are resistant to apoptosis and more likely to undergo senescence after radiation. The latter is associated with a hypersecretory state and may play an important role in shaping the inflammatory state of the TME post-irradiation [3]. However, very little is known about the role of CAFs in regulating the pro-immunogenic effect of radiation, a gap in knowledge that needs to be addressed.

- Endothelial cells (ECs). Single doses of radiation above 8 Gy induce EC apoptosis, and above 15-20 Gy blood flow may be drastically reduced, with the risk of enhancing hypoxia and immunosuppression in the TME. While the acute reduction of blood supply contributes to radiation-induced cancer cell death, it is unknown if this ischemic death in the tumor is immunogenic or tolerogenic. 


\section{BOX 2. Defining clinically relevant mechanisms with existing pre-clinical models.}

Several mouse tumor models are currently available, each with different advantages and limitations in the degree to which they can be used to model the interaction of neoplastic cells, tumor stroma and host immune system of patients [68].

- Syngeneic models: Experimental mouse tumors injected in fully immune competent syngeneic mice are the most frequently used models and have provided the data leading to development of immunotherapy in the clinic. The rapid tumor growth in a microenvironment that does not reproduce the organ of origin and is not shaped during the process of carcinogenesis is a limitation of these models. The advantages are time and cost efficiency, availability of multiple wellcharacterized tumors with varying immunogenicity and mutational burden [69], and the ease of testing the role of a gene in response to treatment by knockdown in the cancer cells or by using a knockout recipient mouse. They can be used to obtain detailed mechanistic information with the caveat that results may be model-specific.

- Genetically-engineered models: These mice develop tumors driven by oncogenic mutations and to some extent reproduce the carcinogenesis process in a more physiological way. Disadvantages are the long time often required for cancer development, the high cost and the low mutation burden. However, rapid progress in technologies to edit genes using CRISP/Cas9 [70] and development of new models reproducing the cold tumor phenotype in patients [35] are very promising. Such models will provide information of which radiation doses and 
fractionation are able to recruit DCs and other immune cells and convert a cold tumor into a hot one.

- Humanized mice: These mice can provide the best opportunity to study the interaction of human tumors with the human immune system. Despite progress in development, several hurdles still exist that limit the usefulness of humanized mice, such as development of human xeno-graft versus host disease and interspecies differences in growth factors and cytokines [68]. Nevertheless, it is possible to engraft immunodeficient mice with a portion of a patient's tumor together with the patient's own peripheral blood mononuclear cells, allowing the analysis of the effect of immunotherapies specific for human cells with the irradiated tumor in an autologous system [71]. Such a system could be used to determine, ex vivo, the most effective radiation regimen and immunotherapy agent combination for each patient, providing a truly personalized in situ vaccination approach. 
BOX 3. A blueprint to establish the partnership between radiotherapy and immunotherapy

Experimental combinations are generally tested first in the metastatic setting in patients with refractory tumors whose immune system has been affected by multiple prior treatments, which renders an effective recruitment of the immune system more difficult and the signal of success more elusive. For example, in a study of radiotherapy and subcutaneous injection of GM-CSF in chemo-resistant metastatic solid tumors, we demonstrated that patients with baseline ratio of neutrophils/lymphocyte $>4$ were significantly less likely to experience abscopal responses from the combination [57]. Below, we list alternative approaches that could allow faster progress to demonstrate if and how radiation can improve responses to immunotherapy.

- Establish a nation-wide registry of abscopal effects in patients who receive radiotherapy during treatment with immune checkpoint inhibitors (ICI). Multiple ICI are approved for a growing number of cancer types. Thus, a systematic analysis of abscopal effects in patients who receive radiation based on clinical need could provide reliable and useful information.

- Expand the use of pre-operative trials (pre-surgical and neoadjuvant) to study the irradiated tumor site. This trial design offers the unique opportunity to determine if a "cold" tumor can be converted into a "hot" one by radiation in patients within only a few weeks.

- Establish a multi-institutional program to identify blood surrogate biomarkers of radiation-induced effects in tumor microenvironment (TME). Circulating DNA, exosomes, microRNAs, cytokines, chemokines, antibodies, 
soluble receptors and lymphocytes should be analyzed in patients receiving radiation alone or in combination with immunotherapy. Identification of shared and neoantigen-specific $\mathrm{T}$ cells in peripheral blood using high-throughput screening techniques [72] would provide needed evidence about the breadth of the anti-tumor response induced by radiation.

\section{- Develop guidelines and biomarkers for immune monitoring in trials testing} radiation and immunotherapy. It is extremely important to gather information about immunological changes associated with response or failure. In addition to parameters normally measured in immunotherapy trials there may be changes specific to radiation that need to be identified. Harmonization of the endpoints will allow comparisons between institutions and trials, enhancing interpretation of data.

- Randomized trials to establish how to best induce abscopal effect in patients with metastatic disease. These trials are required to determine the radiation dose and fractionation, site of radiation, and sequencing with a given ICI that produce the best results. Larger trials should be informed by preclinical data and, whenever possible, by data obtained in pre-surgical studies. They should always be accompanied by correlative studies defined by common guidelines. 


\section{Glossary.}

Abscopal effect: from the latin ab scopus, this term refers to tumor regression seen outside of the field of radiation.

CTLA-4: Cytotoxic T-Lymphocyte-Associated Protein 4. This receptor is expressed by T cells and negatively regulates their ability to respond to antigen and proliferate.

DC: dendritic cell. These cells are the most powerful antigen-presenting cells in the body, capable of activating naïve T cells. DCs uptake antigens from dying cells and can process and present them to $\mathrm{T}$ cells.

HIF-1 $\alpha$ : Hypoxia-Inducible Factor- $1 \alpha$ is a transcription factor that is induced by decrease in available oxygen and regulates the expression of hundreds of genes, many of them cell type specific.

ICI: Immune Checkpoint Inhibitors. This term refers to antibodies blocking receptor that are expressed on $\mathrm{T}$ cells and function as negative regulators of $\mathrm{T}$ cell activation and function. CTLA-4 and PD-1 are immune checkpoints.

PD-1: Programmed Death-1 is a receptor expressed on activated and exhausted T cells. Engagement of PD-1 by its ligands, PDL-1 or PDL-2, which are expressed on antigenpresenting cells decreases T cell activation. PD-1 engagement by PDL-1 expressed on target cells (such as cancer cells) inhibits the function of effector T cells and can induce them to undergo apoptosis.

Radiation dose: energy deposited by ionizing radiation per unit mass, measured in gray (Gy): $1 \mathrm{~Gy}=1 \mathrm{~J} / \mathrm{kg}$. 
STING: Stimulator of Interferon Genes pathway functions to detect the presence of cytosolic DNA and, in response, trigger expression of interferon type I and other inflammatory genes.

TCR: T Cell Receptor. Molecular complex expressed on the surface of T cells and responsible for recognizing fragments of antigen as peptides bound to major histocompatibility complex (MHC) molecules.

TME: Tumor Microenvironment, defines the non-neoplastic cellular environment of a tumor, including blood vessels, immune cells, fibroblasts, extracellular matrix, cytokines, chemokines and other active compounds.

TGF $\beta$ : transforming growth factor $\beta$, is a pleiotropic cytokine with multiple functions in development, tissue homeostasis and inflammation. In the tumor it promotes escape from immune control by inhibition of DC activation and effector differentiation of T cells. 
Click here to download Key Figure KEY FIGURES 1.pptx $\stackrel{ \pm}{ }$

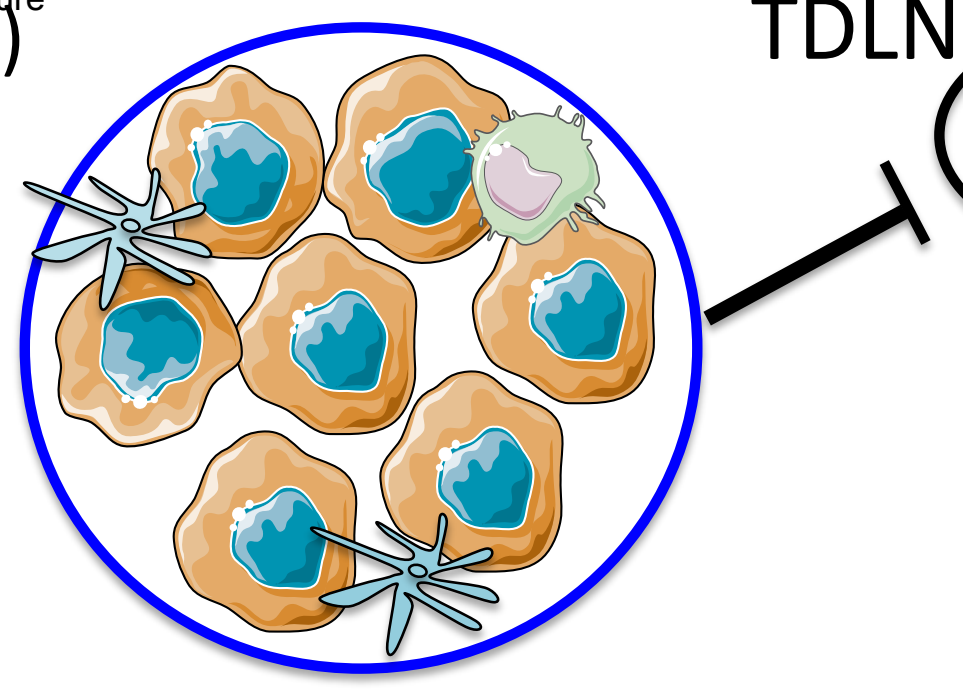

(b)

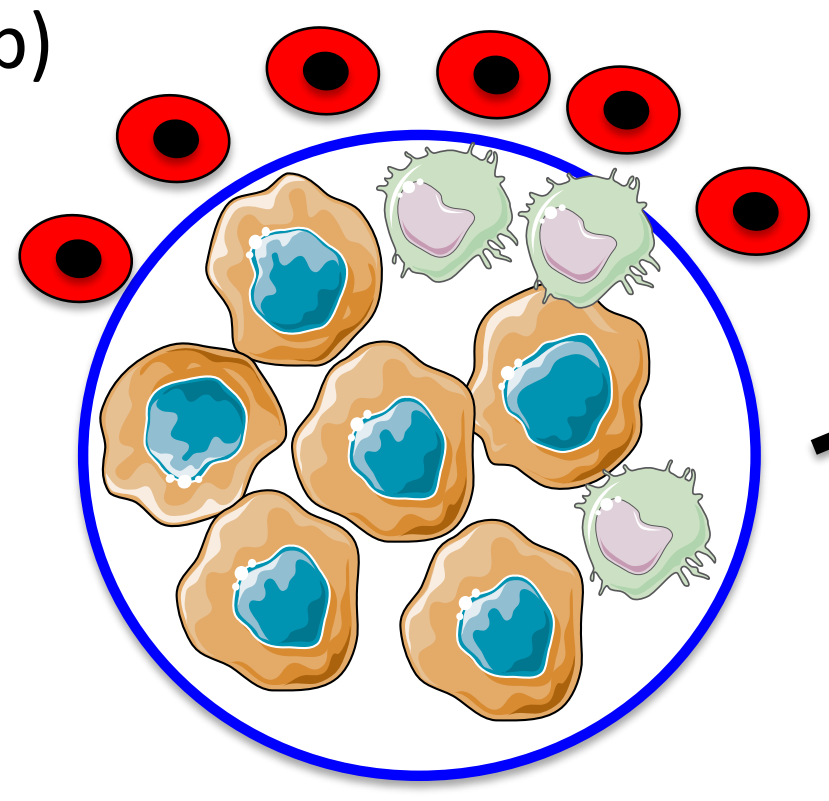

COLD TUMOR

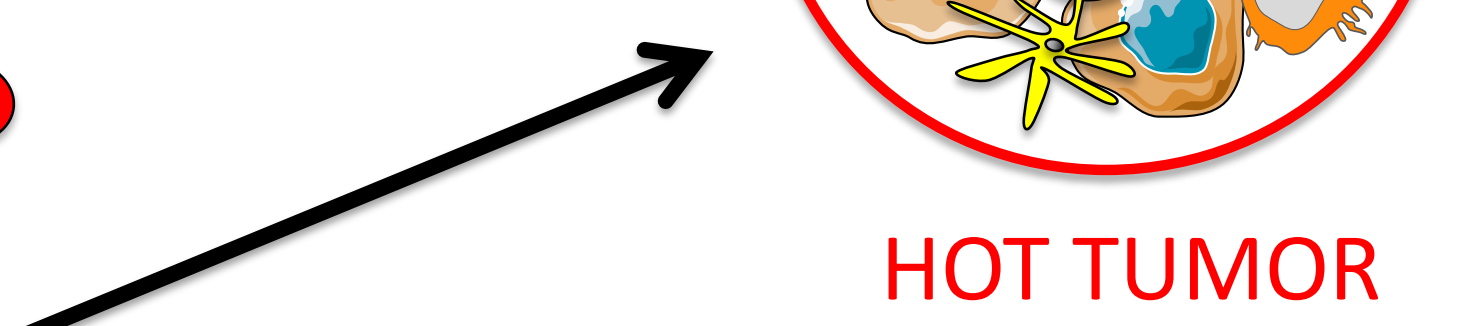

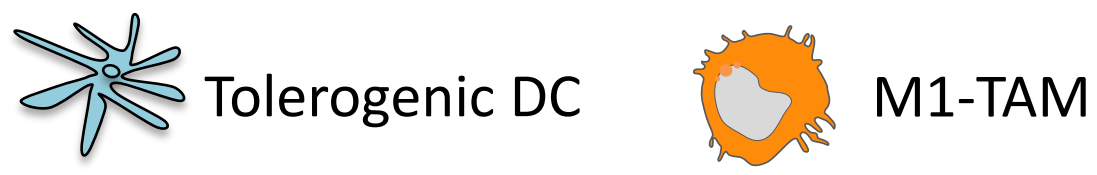

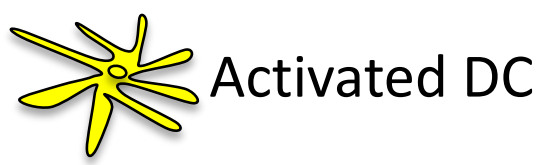

M2-TAM

(- Effector T cell

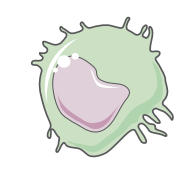

M2-TAM 